\title{
Escapes and absconding from secure psychiatric units
}

\author{
Tim Exworthy, ${ }^{1,2}$ Simon Wilson ${ }^{2,3}$
}

The Psychiatrist (2010), 34, 81-82, doi: 10.1192/pb.bp.108.024372

1Oxleas NHS Foundation Trust Memorial Hospital, London; ${ }^{2}$ Department of Forensic Mental Health Science, Institute of Psychiatry; ${ }^{3}$ South London and Maudsley NHS Foundation Trust, Chaucer Community Mental Health Resource Centre Correspondence to Tim Exworthy (tim.exworthy@kcl.ac.uk)
Summary A prominent radio news programme reported on escapes from secure psychiatric units in the UK and linked that with subsequent offending by psychiatric patients. The report cited did not distinguish between escapes and absconding but, it is argued here, these are very different activities. The acceptable rate both for escapes and absconding from secure psychiatric units is not defined. A certain level of absconding is a consequence of the therapeutic use of leave, which, as part of rehabilitation, is likely to be linked with a reduced reoffending rate post-discharge.

Declaration of interest Both authors work at low secure psychiatric units.

\section{What makes the news?}

On 9 September 2008, BBC Radio 4 reported that 116 people had escaped from medium and low secure psychiatric units during 2007. It was noted that over the same period five individuals had escaped from prison. The report focused on a particular patient who raped a teenage girl after he had 'escaped' from a secure private hospital.

The stigmatising tenor of the report, which was also carried in other media, ${ }^{1}$ is concerning. Linking as it did psychiatric patients with criminal activity does nothing to help keep the risks posed by the mentally ill to the wider community in perspective.

The methodology underlying the report is also highly questionable. No distinction was made between escapes and absconding, or between medium and low secure units. The reported number (116, later reduced to 94) seems to refer to individuals rather than separate episodes..$^{\dagger}$ Independent providers were not included in the survey and not all National Health Service (NHS) trusts featured. The number of 'escapes' ranged from 1 to 15, with the mean of 3.6 per trust. Official statistics recorded 4 escapes from prison and 601 cases of absconding by prisoners during $2006 .^{2}$

\section{Escape or absconding?}

Escapes and absconding are very different activities. Escapes involve a breach of the physical secure perimeter of the building, be it a hospital or a prison. Absconding is generally taken to refer to breaking the conditions of regulated authorised absences from the psychiatric unit (the prison service reserves the term for prisoners gaining their liberty unlawfully while unescorted). Reframing the above in terms of the three aspects of security (physical,

tWe requested clarification on these points from the BBC Radio 4 Today programme, but have never received a response. procedural and relational security), ${ }^{3,4}$ escapes involve breaches of physical and possibly procedural security. By contrast, absconding is a breach of relational and procedural security.

Radio 4's report does raise several important questions. What is an acceptable level for either breach? What is the offending rate while patients are absent without leave as a result of either escaping or absconding (it is very low according to one study ${ }^{5}$ )? In general terms, what is the balance between security and therapy for secure hospital units?

\section{Security v. therapy}

It is clearly a grave concern when someone escapes or absconds from any secure setting and goes on to commit a serious offence. Ostensibly, 'secure units' might be expected to prevent any escape, but the term refers to any hospital unit that is not unlocked and potentially includes both medium and low secure units as well as psychiatric intensive care units. Should the last two have the same success rate in preventing escapes/absconding as the first when, by definition, their level of security is not as high? Is the more important factor the probability of the escape taking place or the likelihood of that person causing harm once he or she has escaped?

Similarly, what level of absconding is acceptable? It could be argued that a unit operating perfectly would have an absconding rate of zero, but this is unlikely to happen in practice. Security itself may be seen in a therapeutic context, both in terms of therapy improving (relational) security, ${ }^{6}$ and safe containment being essential for treatment. $^{6,7}$ The use of leave, too, is inherently therapeutic in secure hospital units and the means by which otherwise sterile risk assessments are gradually tested out in a controlled fashion, something alien to the culture of the closed prison system. Patients are encouraged gradually to 
resume responsibility for their behaviour and they demonstrate that by adhering to the boundaries of leave. The reconviction rate for those leaving secure units is $15 \%$ within 2 years, ${ }^{8}$ compared with $61 \%$ within 2 years for those leaving prison. ${ }^{9}$ Might not the therapeutic use of leave be of central importance to this difference?

Authorised leave should be preceded by an assessment of the level of risks posed by that individual. A very low absconding rate could imply an excessively cautious approach to granting leave and consequently the inappropriate detention of patients who no longer require that degree of security. Conversely, an absconding rate that is too high may suggest a flawed approach to granting leave.

\section{Conclusions}

A wider, informed debate is required about an acceptable level of escape or absconding. Unfortunately, this possibility has been severely hampered by the response of the National Patient Safety Agency to these issues, insisting that the escape (not absconding) of a prisoner transferred to a medium or high secure psychiatric unit should be a 'never event'. ${ }^{10}$ This effectively sets the escape rate at zero for all patients in such units.

We would argue instead for the collection and monitoring of data on these events routinely at a national level. The data would need to incorporate some measure of the 'time at risk', together with a clear idea of the denominator involved (3 cases of absconding from a trust with only 5 secure beds would clearly be more concerning that 20 cases in a trust with 300 secure beds). Statistically outlying performances by individual organisations could then trigger further investigation, just as it happens with surgical mortality data. Such an investigation would then take a more qualitative approach in examining processes and procedures. Even the best risk assessments are probabilistic, so that an adverse event does not necessarily mean the risk assessment had failed or had been done wrongly. Therefore, a unit might get a clean bill of health when its decision-making was shown to have been acceptable, a more sophisticated approach than relying on numbers alone. We would argue that process may be more important than outcome, and examining this rather than over-relying on the raw figures would lead to more benefit for patients overall.

\section{About the authors}

Dr Tim Exworthy is Consultant Forensic Psychiatrist at Memorial Hospital and Visiting Senior Lecturer in Forensic Psychiatry at the Department of Forensic Mental Health Science, Institute of Psychiatry, London; Dr Simon Wilson is Visiting Senior Lecturer in Forensic Psychiatry at the same department and Consultant Forensic Psychiatrist at Chaucer Community Mental Health Resource Centre, London.

\section{References}

1 Booth J, de Bruxelles S. Security fears as 116 mentally ill criminals escape in a year. Times Online 2008; 9 September (http:// www.timesonline.co.uk/tol/news/uk/health/article4714143.ece).

2 Ministry of Justice. Offender management caseload statistics 2006 Statistical Bulletin 2007 (http://www.justice.gov.uk/publications/ offender-management-caseload-stats-2006.htm).

3 Kennedy HG. Therapeutic uses of security: mapping forensic mental health services by stratifying risk. Adv Psychiatr Treat 2002; 8: 433-43.

4 Exworthy T, Gunn J. Taking another tilt at high secure hospitals. The Tilt Report and its consequences for secure psychiatric services. $\mathrm{Br} J$ Psychiatry 2003; 182: 469-71.

5 Gradillas V, Williams A, Walsh E, Fahy T. Do forensic psychiatric inpatient units pose a risk to local communities? J Forens Psychiatry Psychol 2007; 18: 261-5.

6 Adshead G. Three degrees of security: attachment and forensic institutions. Crim Behav Ment Health 2002; 12 (2 suppl): S31-45.

7 Scott PD. Punishment or treatment: prison or hospital? BMJ 1970; 2 167-9.

8 Maden A, Scott F, Burnett R, Lewis GH, Skapinakis P. Offending in psychiatric patients after discharge from medium secure units: prospective national cohort study. BMJ 2004; 328: 1534-5.

9 National Offender Management Service. Offender Management Caseload Statistics 2004, England and Wales. Home Office, 2005.

10 National Patient Safety Agency. Never Events. Framework 2009/10. Process and Action for Primary Care Trusts 2009/10. National Reporting and Learning Service, National Patient Safety Agency, 2009. 